\title{
Kinematic Alignment and Bonding of Silicon Mirrors for High- Resolution Astronomical X-ray Optics
}

\author{
Kai-Wing Chan ${ }^{1, \mathrm{a}, \mathrm{c}}$, James R. Mazzarella ${ }^{\mathrm{b}, \mathrm{c}}$, Timo T. Saha ${ }^{\mathrm{c}}$, William W. Zhang ${ }^{\mathrm{c}}$, Ryan S. \\ McClelland $^{\mathrm{b}, \mathrm{c}}$, Michael P. Biskach ${ }^{\mathrm{b}, \mathrm{c}}$, Raul E. Riveros ${ }^{\mathrm{a}, \mathrm{c}}$, Kim D. Allgood ${ }^{\mathrm{b}, \mathrm{c}}$, John D. Kearney ${ }^{\mathrm{b}, \mathrm{c}}$, \\ Marton V. Sharpe ${ }^{\mathrm{b}, \mathrm{c}}$, Michal Hlinka ${ }^{\mathrm{b}, \mathrm{c}}$, Ai Numata ${ }^{\mathrm{b}, \mathrm{c}}$ \\ ${ }^{a}$ Center for Research and Exploration in Space Science and Technology \& University of \\ Maryland, Baltimore County, Baltimore, MD 21250 \\ ${ }^{\mathrm{b}}$ Stinger Ghaffarian Technologies, Inc., Greenbelt, MD 20770 \\ 'NASA/Goddard Space Flight Center, Greenbelt, MD 20771
}

\begin{abstract}
Optics for the next generation's high-resolution, high throughput x-ray telescope requires fabrication of well-formed lightweight mirror segments and their integration at arc-second precision. Recent advances in the fabrication of silicon mirrors developed at NASA/Goddard prompted us to develop a new method of mirror alignment and integration. In this method, stiff silicon mirrors are aligned quasi-kinematically and are bonded in an interlocking fashion to produce a "meta-shell" with large collective area. We address issues of aligning and bonding mirrors with this method and show a recent result of 4 seconds-of-arc for a single pair of mirrors tested at soft x-rays.
\end{abstract}

Keywords: X-ray optics, lightweight mirrors, segmented mirrors, silicon mirrors, mirror alignment, mirror bonding

\section{INTRODUCTION}

Current concept of high throughput $\mathrm{x}$-ray telescopes invariably involves integrating a large number of thin mirror segments into a very compact telescope ${ }^{1}$. The most practical approach for very large $\mathrm{x}$-ray telescopes presently is to concentrically nest thin mirror segments together to form a large telescope. Mirrors of various approximations of the Wolter type-I optics with nested concentric shells were used in missions such as ASCA ${ }^{2}$, Suzaku ${ }^{3}$, NuSTAR ${ }^{4,5}$, and Hitomi $^{6}$. It is also the approach taken by the Athena ${ }^{7}$ project. (We will not discuss the full-shell mirrors in this paper. Full shell mirrors have been used in many missions such as Chandra and XMM-Newton. It is also used in an upcoming mission e-Rosita. Full shell mirror has its own characteristics and merits---especially when the size is small, and mass and area are not important constraints. It is, however, difficult and expensive to scale the production of full shell mirrors to make large and lightweight optics for the next generation of $\mathrm{x}$-ray telescopes.) The advantage of thin mirror segmented optics is that many mirror shells can be integrated into compact packages to achieve a very large effective area. The segmented approach is also scalable. The challenge of such effort usually is to improve the optics resolution, as both distortion of each of these easily-deformed thin mirrors and the misalignments between them invariably worsen the telescope's resolution.

In general, the technology in building high-resolution segmented x-ray telescope from thin substrates can be divided into three areas: (1) the fabrication of precise mirror substrates; (2) the subsequent processes of delicate aligning and mounting the thin-shell mirrors without introducing additional unacceptable distortion; (3) alignment and integration of the modules or meta-shells into a full telescope. In this paper, we will concentrate on the alignment and bonding of segmented mirrors. The objective is to build a sub-structure which we called a "meta-shell", in which the external telescope frame is nearly completely eliminated. The fabrication process of substrates, made of single crystal silicon $^{8}$, and the current status ${ }^{9}$ is reported in a separate paper by Riveros, et al., in these proceedings. The optical design of the telescope ${ }^{10}$ and structural analysis of such meta-shell ${ }^{11}$ is detailed in other papers by Saha et al., and McClelland, et al., in reference to the STAR-X mission concept, which is a proposal submitted in December 2016, in response to a NASA Explorer call. These technologies, the requirements for astronomical x-ray optics in general, and

${ }^{1}$ Kai-Wing.Chan-1@nasa.gov 
how they can be used to implement mission concept for future large, high resolution astronomical x-ray telescope, is reviewed $^{12}$ in a paper by Zhang, et al.

Integration of mirrors with thin (typically $0.2-0.5 \mathrm{~mm}$ ), segmented, substrates can be broadly divided into two camps of approaches. One approach is to fabricate mirror segments precisely to meet the telescope requirements, and mount them into telescope housings independently of each other with negligible distortion ${ }^{13}$. In this case, the only coupling between the individual mirror is through the housing/module they commonly reside in. An alternative approach, which can be called the "stacking mirror" method, is to attach mirrors of the next shell directly onto mirrors already integrated, instead of onto an external structure. The non-stacking mirror method was used on previous missions such as ASCA, Suzaku, and Hitomi, while the stacking mirror approach was taken successfully by NuSTAR. ESA's mission, Athena, takes the stacking concept in another direction in that flat mirrors are forced, bent and fixed onto the existing stack.

Our group at NASA/Goddard Space Flight Center (GSFC) has been actively pursuing both approaches. The advantage of non-stacking mounting is that mounting distortion can be independently managed and there is virtually no stack-up error. This approach, however, places very stringent requirements on the figure of the individual substrates, the method of alignment and mounting, and the requirement of the external structure. We also explore the stacking approach. For the stacking approach to work without stack-up error, precise spacers between mirrors have to be made and a mean to reset any error from the existing stack must be developed. For such a stack to achieve, say, the level of Chandra's resolution of 0.5 " half power diameter (HPD, or half energy width, HEW), significant improvement has to be made, however, to get from NuSTAR's $\sim 1$ arc-minute resolution to the arc-second level.

Because of the advance in the fabrication of silicon mirrors, we revisited the stacking approach which can result in a more homogeneous all-silicon, highly thermally conductive structure. Since the outer layers of mirrors are built upon the inner layers, the mirrors themselves provide the mechanical structure and the need of an external structural support can be minimized. Our goal in this approach is to advance bonding precision to arc-second level by eliminating mirror distortion due to mirror mounting and remove the stack-up error by resetting the mirror position and orientation precisely for each mirror segment. In this way, we aim to develop technology in building up meta-shells with high precision and high throughput.

It should be noted at, as a matter of principle, our approach is to produce the more precise mirror segments that meet specified requirements and integrate them into a telescope without further distortion. That is, the mirrors should be aligned and integrated without using the alignment or integration step to "correct" for any figure error of the mirrors. It is our belief that the technologies (mirror fabrication, alignment, integration) of future large telescopes can be more independently developed, and the implementation of various processes to build the telescope need not be substantially linked together. We take it as our working principle. As such, methods such as that used in Athena is not considered. At this point, we also do not consider active optics.

\subsection{Four-point mounting of silicon mirrors and integration into meta-shell}

We have reported on the results of the non-stacking mirror approach in the past. In the so-called edge bonding ${ }^{14}$, each individual mirror is aligned individually and is bonded onto the telescope housing at the mirror's edges, where inplane forces do not distort the mirrors locally as easily as forces perpendicular to the mirror's plane. The substrates we use for building such telescope are made of glass. These thin glass sheets are thermally slumped onto accurately figured mandrels. Each mirror is then aligned with a precision hexapod before it is bonded onto a module housing with epoxy. This method is simple and the alignment and bonding can be made quite accurately, to within $\sim 5$ ".

The same alignment and bonding method, in principle, can be applied to silicon mirror. However, the edge-bonding method encounters several difficulties which forbid it from meeting the higher demand in precision in alignment. For example, even though the hexapod used in the alignment is quite precise (repeatable to $\sim 1$ "), the epoxy that is used to join the mirror to the module housing builds up stress when the epoxy is cured. This causes a generally unpredictable mirror misalignment of a few seconds-of-arc when the mirror is finally handed off to the structure.

With the advance of the more precise silicon mirror, we revisited the alignment concept. Silicon is also stiffer and is therefore even less susceptible to local distortion during bonding. Summarizing the lessons learned, we determined that a kinematic mounting method where a mirror is in good point contact to its support is a way to address the alignment and bonding issue. 
In the following, we will discuss a particular implementation of this method for bonding of individual mirrors, the 4point bonding method. In this method, we start with a mirror of sufficient optical quality. In our case, it is the polished mono-crystalline silicon mirror. It is placed, reflecting surface facing down, onto 4 precisely placed supports. The "heights" (actually the radial positions) of the supports, which we called spacers, are accurately machined. The 4 spacers, together with an axial stop and an azimuthal stop, uniquely determines the orientation and position of the paraboloidal or hyperboloidal mirrors in space. The mirrors are placed properly by controlled vibration, which sets the mirror into its statically determined orientation by relaxing the effect of friction. Sub-aperture measurements of the mirror in an optical beam are taken, from the centers of which the focusing quality of the alignment is assessed. The spacers' radial height are corrected until the alignment is correct. The mirror is then bonded onto the spacers with a small amount of adhesive. For the purpose of applying the adhesive, the mirror is removed. This procedure introduces no additional error as long as the alignment can be maintained after the mirror is replaced, even in the presence of the adhesive. Subsequently, 4 new spacers can then be attached onto the back of the bonded mirror, and the process can then be repeated for the integration of mirrors in the next shell.

For the next shell, we can improve the structural strength of the telescope by integrating the mirrors in an interlocking fashion, similar to laying bricks to build a brick wall. The interlocking approach does not benefit from the modularity of the telescope in the traditional sense but it does produce a full shell which is a single unit that can be integrated into a whole telescope. The immediate product is multi-shell mirror consisting of many mirror segments and having a much larger effective area compared to a thick polished shell such as that used in Chandra. We call it a "meta-shell." As already pointed out, the meta-shell mirror method eliminates many problems with external structure and minimize the mass of the telescope. This approach has the following distinct advantages: (1) Lightweight structure; (2) High overall mechanical strength of the integrated telescope; (3) Nearly homogeneous structure; (4) Simpler, full-shell, alignment. In the meta-shell method, besides the inner core, the only additional components other than the mirrors themselves are the joining members between mirrors. Compared to the approach that uses external module housing, the meta-shell approach adds very little mass to the mirrors. Even x-ray telescope reputed to be lightweight has $\sim 50 \%$ of the mass in the housing (for example, the Hitomi telescopes had more than half of it mass in the housings.) Moreover, once integrated, a meta-shell is relatively stiff due to the interlocking mirrors, and the entire structure is more or less homogeneous. The only foreign materials are the thin $(\sim 15 \mathrm{~nm})$ metallic coating such as iridium and the small amount of adhesive that is used to join the mirrors and spacers together. The spacers can be made of silicon, too (even though it is not presently). See the paper by McClelland, et al., for details of the current concept.

The challenge of using the stacking approach to build a meta-shell, as outlined above, is therefore in maintaining the precision in the alignment of mirrors, the adjustment of spacer heights, and the minimization of misalignment from bonding.

\section{FOUR-POINT KINEMATIC MOUNTING OF NEAR CYLINDRICAL MIRROR SEGMENTS}

For the alignment and building up of mirrors, unlike that used in the optics for NuSTAR, we choose to use a 4-point configuration ( 2 points axially at only 2 azimuthal positions) instead of bonding mirrors at the full axial length. Clearly, bonding a mirror at full axial lengths over-constrains the mirror unless the axial profiles of the bonding elements (and the epoxy thickness) match that of the mirror at those azimuths. This is a stringent requirement for high resolution telescope at arc-second precision (NuSTAR's angular resolution is 1 arc-minute). Joining the mirrors only at two, but not more, azimuthal positions also minimizes the entrance obscuration and enhances the telescope throughput. Most importantly, a mirror simply-supported at 4 points (with extra constraints for axial translation and roll degrees of freedom, see below) also statically determines the orientation of the mirror. That is, a mounting scheme as such ensures that all 4 support points, and only those 4 points, are in contact with the mirror surface and it does not further distort the mirror besides that of gravity sag.

\subsection{The "4-piont mount" concept}

Since a 4-point kinematic support is less common (most kinematic mounts of optics are made with 3 supports), it may be instructive to clarify our choice here. This choice is more natural for the quasi-cylindrical nature of the $\mathrm{x}$-ray mirror in Wolter-type configuration.

For the mounting of the thin segmented mirrors, we want to align the mirror in a simply-support fashion (no moments at the joints) so that the orientation of the mirror is determined by its own optically precise shape. This calls for a 
kinematic mounting. For example, for a flat, the surface can be uniquely defined with a simple 3-point support that determines the direction of the plane as well as its overall "height" in the gravity field. A flat of finite size is still free in the remaining 3 degrees of freedom (DoF): it is allowed to "slide" in the tangential horizontal plane, as well as to rotate in that plane about the vertical axis. These operations, however, are invariant as far as the surface is concerned. Translation in the plane's tangential directions and rotation about its own normal direction are invariant operations for a large planar surface, and therefore has no consequence in the reflection of the light off it. Additional constraints, however, are needed to completely determine the orientation and position of the flat, when it is taken as a finite solid. This can be accomplished in a number of ways, by imposing more constraints of more DoF at one or more points.

For a cylindrical surface or a section of it, we want to take advantage of the symmetry that the surface is defined for the kinematic mount. (In the discussion in this paper, we will use the following coordinate system for any cylindrical or near-cylindrical surfaces: The axis is along the $z$ axis, which is horizontal; the inner surface/reflecting surface is facing down towards $-x$; the tangential direction is approximately in the $y$ direction, which is horizontal in the gravity field.) The symmetry of the surface is that it is defined by 4 parameters (for example, the direction of the cylindrical axis (a unit vector, $n_{z}$ ), and the coordinates of the axis intercepting, say, the $y-z$ plane). Rotation about it and translation of the cylinder along its axis are invariant operations for the cylindrical surface. The constraints from the 4 points therefore provide 4 equations to solve for 4 parameters that uniquely define the cylindrical surface. To completely determine the orientation and position of the cylinder as a solid body, two additional constraints are neede to stop the cylinder from sliding along the axial direction and from rotating about its own axis. These can be implement in a number of ways, for example, by constraining all $3 \mathrm{DoF}$ at one of the points.

The case for a section of conical surface with a given semi-cone angle is similar to that of a cylindrical surface. The conical surface has less symmetry than that of a cylinder, however. It is defined by 5 parameters (for example, the direction of the axis of the cone ( 2 parameters), and the coordinate of the apex of the cone ( 3 parameters)). The rotation about the cone's axis $\left(\theta_{z}\right)$, again, is invariant, but the translation along the $z$ axis is strictly not invariant. However, for grazing incidence $\mathrm{x}$-ray optics, the cone angle is typically very small $\left(<1^{\circ}\right)$. The translation is therefore approximately invariant. We can take advantage of this insensitivity to axial translation and ignore any small translation along the axis. So, in practice, a surface simply-supported at 4 points $\left(x, y_{i}, z_{i}\right)$, where $i=1,2$, and $x$ is arbitrary ( $x$ for the 4 points even need to be the same), will completely constrain the quasi-cylindrical surface. Additional axial and azimuthal stops will hold the solid mirror statically in a quasi-kinematic way that is suitable for grazing incidence $\mathrm{x}$ ray segmented optics. One can interpret this mount physically as follows. A pair of spacers at the same axial position $z_{i}$ determines the "pitch" angle ( $\theta_{y}$, rotation about the $y$ axis) for the mirror. Raising or lowering the height $(x)$ of the pair of spacers changes primarily the angle of incidence of the incoming ray. Adjustment of the height of a single spacer changes its "yaw" angle $\left(\theta_{x}\right.$, rotation about the vertical $x$ axis). In fact, raising the height of one is very approximately equivalent to lower the height of its diagonal spacer.

Evidently, the surface of revolution generated from a parabola or hyperbola---those prescribed in a Wolter type mirror design---has the symmetry that is similar to that of a cone, requiring also 5 parameters to define its surface. The same 4-point support with side pins to stop the solid mirror from sliding off in the axial direction and rotating off about its axis will therefore be adequate to mount the surface uniquely, and this is the choice of our mounting scheme.

\subsection{The integration of mirrors into multiple shell or meta-shells}

As described above, once a single mirror in a single shell is properly mounted, the other shells can be integrated in an interlocking fashion to form a rigid, lightweight, meta-shell that is well defined to be aligned into a compact telescope. To build the stack of mirrors, we choose an "inside-out" approach in which the $(n+1)$-the mirror is added onto the back of the mirror in the $n$-th shell, with the telescope's optical axis horizontal. The mirrors will be azimuthally staggered so that a ring of the mirrors are integrated together like that of a brick wall. The mounting of each mirrors is independent. The laying of the mirror shells is therefore quite straightforward provided that the following challenges are met.

First of all, surface-to-surface variation between mirror shells need to be very precise. With the typical dimension for the mirror under consideration ( $100 \mathrm{~mm}$ in linear dimension, $\sim 1 \mathrm{~m}$ in mirror diameter, and $\sim 10 \mathrm{~m}$ focal length $)$, the accuracy of spacer height is required to be $\sim 0.1 \mu \mathrm{m}$ for arc-second $\mathrm{x}$-ray optics. That is, the sum of spacer height and mirror thickness is required to be accurate to that level. The variation of the mirror thickness and the spacer thickness 
can be brought to within several $\mu \mathrm{m}$. Further processing is therefore needed to bring the spacer height, $z_{i}$, to $0.1 \mu \mathrm{m}$ accuracy. Achieving this precision economically and efficiently is essential for this scheme to work

Secondly, the adhesive used to bond a mirror to its spacers will mis-align the mirror, especially when the epxoy change upon curing. How to preserve that sub- $\mu \mathrm{m}$ accuracy if we bond the mirror with adhesive is another challenge. In the sections below, we will describe the practical issues in the implementation of the 4-point scheme and results from the experimental procedure.

\section{IMPLEMENTATION AND TESTING OF 4-POINT ALIGNMENT AND BONDING}

To demonstrate that the 4-point alignment and bonding scheme, we have devised a platform for a single pair of mirror. The concept is shown in Figure 1. The platform is made of silicon for thermal purpose. Eight spacers, currently made of stainless steel, are attached onto the solid mount, four for each mirror. Specifically, the mirror is $100 \mathrm{~mm}$ long. Its radius of curvature is $156 \mathrm{~mm}$ and spans $30^{\circ}$ azimuthally. The design focal length of the mirror pair is $8.4 \mathrm{~m}$. The spacers are separated in the axial direction by $56 \mathrm{~mm}$ to minimize the gravity distortion of the mirror when it is simplysupported. The spacers are $3 \mathrm{~mm}$ in diameter.

The base itself is seated on a stage stack. The mount is, again, kinematic. The kinematic mount is realized by having three steel balls, which are attached to the bottom of the silicon base, sitting on 3 grooves. Each of the grooves constrains, in addition to the vertical DoF (in $x$ ), one DoF in the $y-z$ plane. The repeatability of the relatively solid silicon mount is very good (error negligible).

In the section, we will discuss the major steps that are needed to align and mount the mirrors precisely. We will address the following questions: (1) How precisely can a mirror be placed onto 4 spacers? Will friction between the spacers and the mirror prevent the mirror from settling onto its mathematically determined position as described in Section 2 ?

(2) How do we measure the resulting focusing quality of the mirror to sufficient precision? (3) Given an optimal alignment of a mirror, which is largely determined by the orientation of the mirror with respect to the incoming light, how is the second mirror co-align to the first? How is it best to handle the near degeneracy of the pitch of a mirror and its focused image position? (4) How should the spacer heights be adjusted precisely and efficiently? (5) How are the mirror bonded accurately?

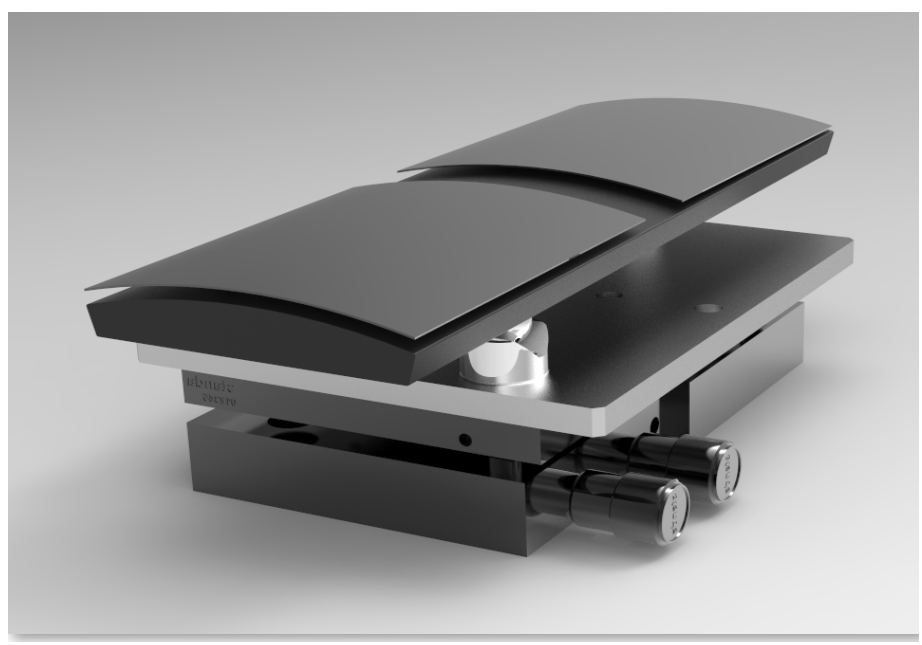

Figure 1. Concept of testing a pair of mirrors mounted on a base with the 4-point mount. The base is a cylinder made of silicon and has a radius of curvature compatible with that of the mirrors. Eight spacers (hidden by the mirrors) are currently made of stainless steel but can be made of silicon to build a complete unit with homogeneous material. Spacer heights are pre-set in 4 different prescriptions.

\subsection{Precision of placement a single mirror segment in 4-point mount}

Needless to say, without additional assistance, friction between the spacers and the mirror will prevent the mirror from settling onto its mathematically determined position. With the aforementioned mirror dimensions, the angles between 
the mirror surface to the horizontal plane at the spacer locations are simply too small to overcome friction, despite the fact that the mirrors are smooth. The mirror is also symmetric azimuthally such that there is little tendency for the mirror to slide. To settle the mirror into place, we currently employ a small vibrational motor, which can be coupled to the silicon base temporarily. The simple set up is shown in Figure 2.

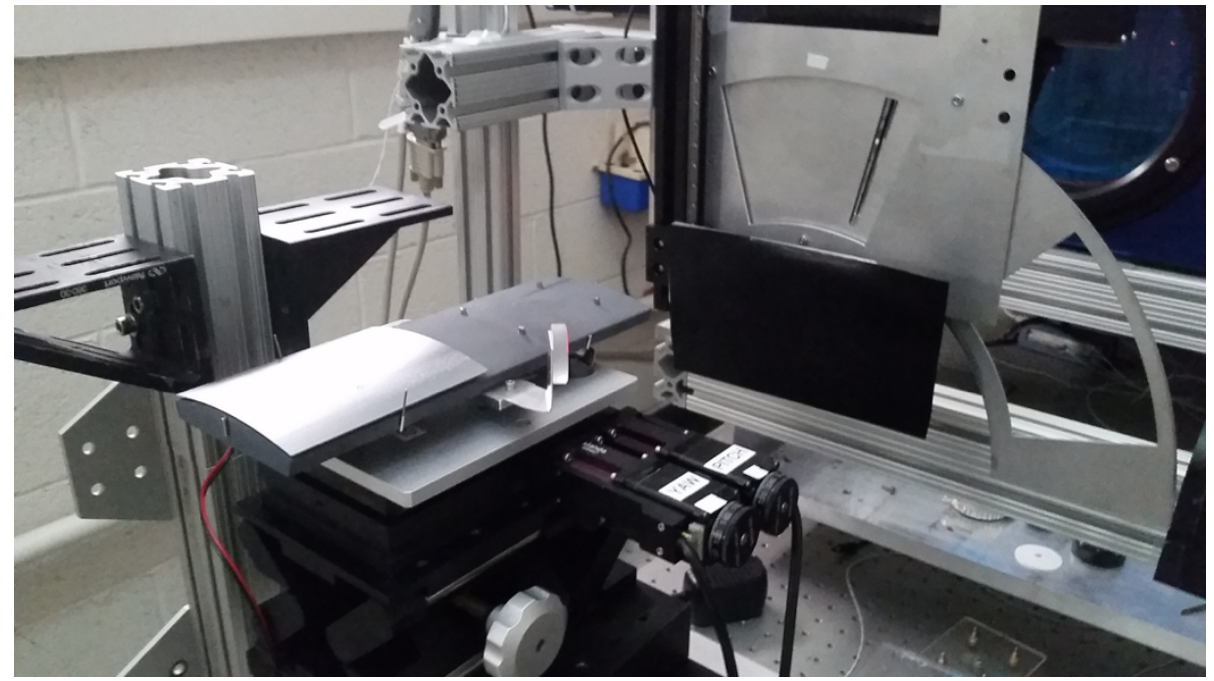

Figure 2. Repeatability of self-relaxing alignment of mirrors onto a 4-point mount. A parallel beam is illuminated onto the mirror sitting on the 4 spacers, through either a completely open aperture or a movable slit. The reflected light, which is also highly diffracted due to the small grazing incidence, is captured on a camera (not shown). The distribution of the centroids of those images determined the focusing of the mirrors.

This simple process allows the mirror to settle onto its natural relaxed state in which all 4 points are in contact. The state of mirror is also indirectly confirmed from the observation that the mirror can be repeatedly placed precisely in the same orientation. Measurement of the focus shows that individual azimuthal sectors have the image centroid within 1". Typical rms variation of the center of the sub-aperture images is about 0.2 " in a 11-point measurement. The process does depend on how the mirror is agitated into place, however. Only a mild vibration is effective. Further quantification of the vibration's amplitude and modes is being studied to determine the limits of the process.

\subsection{Measurement of focus of aligned mirrors}
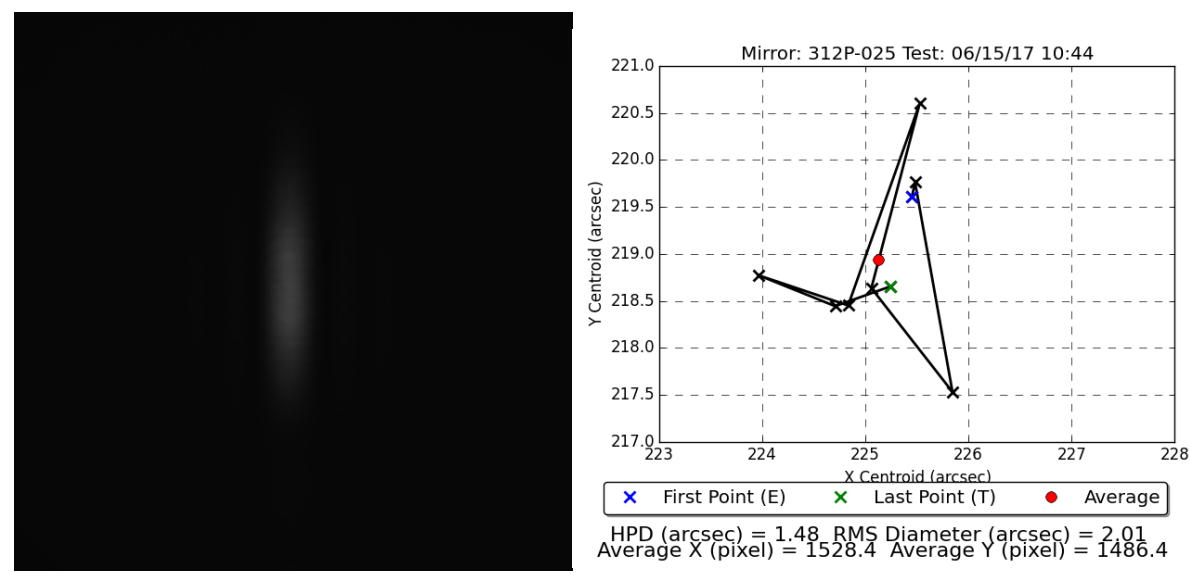

$\begin{array}{rl}\mathrm{HPD}(\operatorname{arcsec})=1.48 & \mathrm{RMS} \text { Diameter }(\text { arcsec) })=2.01 \\ \text { Average } X(\text { pixel) } & =1528.4\end{array}$

Figure 3. Example of optical measurements of image from individual azimuthal sector (left panel) and overall distribution of the image centers showing the focusing of mirrors. Typical error in single point measurement is approximately 1" and multiple measurements are used to reduce the statistical error to sub-arc-second. Overall error in the determination of the center of the full image is about 0.2 ". 
To measure the perfection of alignment, the mirrors are placed, on axis, in a parallel beam and its focused image is capture on a camera placed at the focal length of the mirrors. The sub-aperture measurements were made with a movable mask. The imager is an optical charge-coupled device with $12 \mu \mathrm{m}$ pixels. (At $\mathrm{f}=8.4 \mathrm{~m}$, the resolution limited by the pixel size is about 0.3 " /pixel.) The alignment of mirrors either from single or double reflection is determined from the distribution of centroid of images from individual sub-apertures in the azimuthal direction. Each sub-aperture exposure produces a highly diffracted image in the radial direction. The image center of a sub-aperture image represents the effective direction of that section of mirror surface at that azimuth. Even though the images are highly diffracted, their centroids, however, can be determined to better than 1" when the image background is properly subtracted. Examples are shown in Figure 3.

\subsection{Co-alignment of mirrors for 2-reflections}

The focusing of the optimally aligned mirror is determined by the orientation of the mirror with respect to the incoming beam. In addition, the image is constrained to be at the position determined by the optical axis for on-axis illumination. This constraint also determines the co-alignment of the mirrors in the primary and secondary stage. The alignment is accurate only when both the orientation and its position with respect to the optical axis are proper simultaneously. The optical axis can readily be defined by a round aperture through which the parallel beam goes through. For this purpose, we use a beam with a diameter of $250 \mathrm{~mm}$, which is sufficient to cover both the mirror and their optical axis position.

The on-axis condition is set with the 2-axis tip/tilt stages on which the silicon base is mounted on. The round aperture that registers the optical axis is adjusted with an X-Y translational stage driven with micrometers. We should note that, to meet the position requirement of the optical axis, only mild tolerances of $\sim 5 \mu \mathrm{m}$ is needed (corresponding to 0.1 " at $f=8.4 \mathrm{~m}$ ) for the present precision requirement. The size of the hole is not critical, but it is chosen to be $\sim 3$ $\mathrm{mm}$, to minimize the size of the diffracted Airy disc.

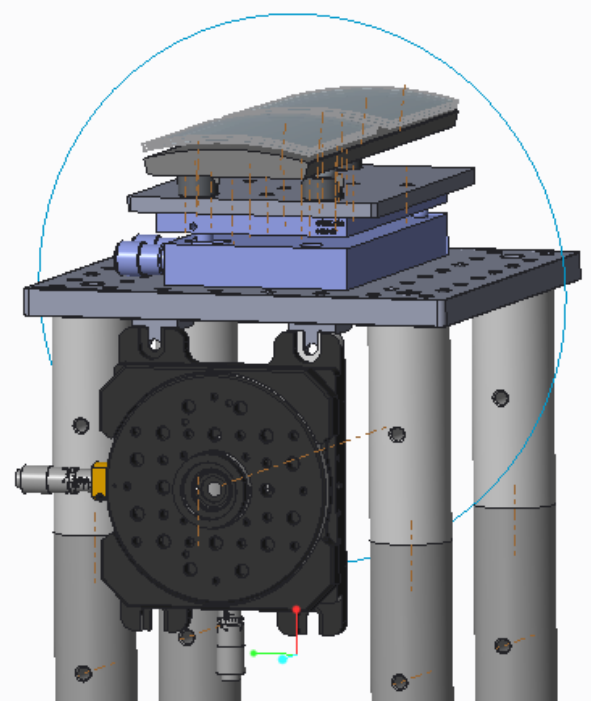

Figure 4. Light from the parallel beam goes through a simple round hole is used to register the optical axis of the mirrors at their respective focal distances. The size of the hole is chosen to minimize diffracted Airy disc.

\subsection{Adjustment of spacer-heights}

Once the quality of the alignment is assessed and its errors determined, the radial heights of the 4 spacers will needed to be adjusted to put the mirrors into their optimal orientation and positions. As described in Section 2, heights of the 4 spacers $\left(x_{i}, i=1, \ldots, 4\right.$, at coordinates $\left.\left(y_{i}, z_{i}\right)\right)$ completely determined the orientation of the mirror and the location of its image on the focal plane. One may intuitively consider adjusting one of the 4 spacers to adjust the yaw angle $\left(\theta_{x}\right.$, rotation about the vertical $x$ axis) of the mirror, and a pair of spacers at the same axial mirror location $(z)$ simultaneously to adjust the pitch angle ( $\theta_{y}$, rotation about the horizontal $y$ axis). Similarly, the image position in the mirror radial direction can easily accomplished by adjusting all 4 spacers simultaneously, and that in the tangential direction is adjusted by working on a pair of spacers at the same azimuthal mirror locations $(y)$ simultaneously. As we shall see, 
currently, we only employ means to reduce spacer heights, the choice of which spacers to be worked on is further limited. In particular, there is no provision to move the mirror radially outward, since that requires increasing the spacer heights of all 4 spacers simultaneously. The spacers are therefore chosen to be biased towards larger lengths to begin with.

We should also note that the spacer heights were originally set only to rough mechanical tolerances (a few to $\sim 10$ $\mu \mathrm{m})$. They have to be corrected to $\sim 0.05 \mu \mathrm{m}$ in order to meet the orientation requirement of arc-second optics. In contrast, the position requirement, as mentioned above, is much more moderate (again, $\sim 5 \mu \mathrm{m}$ corresponds to 0.1 " at $f=8.4 \mathrm{~m}$ ). That is one of the reason why the optical axis can be set sufficiently precisely with mechanical tolerances. In practice, only the relative heights (that is, 3 out of the 4 spacers) are required to be adjusted, with little loss of light collecting efficiency. (The tolerance is $\sim a \eta \sin (\alpha) \sim a R /(4 f)$, where $a$ is the axial length of the mirror, $\alpha$ the angle of incidence, $R$ the radius of the mirror, $f$ is the focal length, and $\eta$ is the fractional tolerance of interception of light. For $\eta=5 \%$, the height tolerance is about $40 \mu \mathrm{m}$, which can be set mechanically.)

Currently, we use very simple and traditional polishing to achieve this spacer height adjustment. In this procedure, the silicon mounting block, which is on kinematic mount, is removed from the set up and placed under a polisher. The removal rate of the polishing was pre-calibrated so that the target removal can be achieved by timing. Such polishing is not entirely straightforward, as the loading, history, surface condition, etc., of the polisher and, to some extent, of the spacer's surface, influence the material removal rate. Nevertheless, with a fine polishing grit, such simple process can achieve $\sim 0.05 \mu \mathrm{m}$ precision needed.

\subsection{Bonding of mirror on 4 spacers}

Among the various steps in the alignment and bonding processes, the effect of adhesive is perhaps the hardest to control. In particular, the following potential issues are to be addressed: (1) Will the surface tension of the adhesive forbid the mirror to relax into its natural figure and position (different from when there the adhesive is not present)? (2) Will there be any significant variation (a small fraction of a $\mu \mathrm{m}$ for the aforementioned dimensions of mirrors) of the bond thickness? (3) What is the impact of the curing of the adhesive? (4) What is the long-term stability of such bonding?

Our lessons learned from previous bonding, using epoxy with a different bonding configuration, indicate that the nonzero bond line is critical to the stability of mirror against epoxy curing, as it is the nature of epoxy to undergo dimensional change when it is being cured. We have experimented bonding with different adhesives with different viscosities, surface tension, and curing times, with different bonding procedures, with accelerated heat-curing, and with additional loading at the bond points to reduce the local bond thickness. Generally, better and more stable bonding can be obtained with the application of a smaller amount of epoxy.

The 4-point configuration opens up a new opportunity to achieve small, or even negligible, bond line. In the 4-point mount, the mirror simply sinks itself into the fluid epoxy uniformly, and attains its natural orientation once again. In fact, the fluid epoxy may even aid that process. The process was significantly improved when "crowned-top" spacers were used. A crowned top spacer is one in which the top surface is rounded, so that it contacts the mirror at a single point. It appears to solve two of the problems mentioned above. First, there is essentially no variation of the epoxy thickness, as the mirror remains in contact, at that single point, after the epoxy is introduced. This is verified both by optical measurement of the mirror orientation (again, sensitive to $\sim 0.05 \mu \mathrm{m}$ differentials between spacers) and by testing the electrical conductivity/resistance through the joints before and after the bonding. Moreover, postmortem examination of the epoxy ring removed from the mirror and spacer shows that the epoxy ring is indeed one with a point hole at the middle, indicating zero epoxy bond thickness at the contact point.

Presently, the consistency of the bonding operation is satisfactory, but not perfect. Some of the difficulties are operational, such as the mirror can slide when it begins to sink down on the bead of epoxy fluid. Nevertheless, it is workable and the "rss" error of the bonding process is approximately \pm 0.7 ". It typically changes a 1.5 " focusing into $1.5 " \pm 0.2$ " (HPD).

The various processes, their status and comments are summarized in Table 1. 


\begin{tabular}{|c|c|c|c|}
\hline Process & Basic process & Current Status & Issues and Development \\
\hline $\begin{array}{l}\text { [1] Placing mirror on } 4- \\
\text { Spacer }\end{array}$ & $\begin{array}{l}\text { - Simply supported at } 4 \\
\text { points } \\
\text { - Vibrated into position } \\
\text { - Mirror is kinematically } \\
\text { supported } \\
\text { - "Roll" and z-axial } \\
\text { DOF constrained with } \\
\text { stop-pins }\end{array}$ & $\begin{array}{l}\text { - Placement repeatable to } \\
<1 \text { " point-by-point } \\
\text { - Needed amplitude of } \\
\text { "agitation" depends } \\
\text { somewhat on the mass of } \\
\text { the mirror }\end{array}$ & $\begin{array}{l}\text { - Currently no } \\
\text { fundamental issues. } \\
\text { - Need a new method to } \\
\text { shake mirror into place } \\
\text { for meta-shell } \\
\text { integration }\end{array}$ \\
\hline $\begin{array}{l}\text { [2] Measurement of } \\
\text { focusing }\end{array}$ & $\begin{array}{l}\text { - Sub-aperture } \\
\text { measurement of mirror } \\
\text { centroid in optical beam }\end{array}$ & $\begin{array}{l}\text { - Measurement repeatable } \\
\text { to } \sim 1 " \\
\text { - Overall centroid } \\
\text { determination } \sim 0.2 " \\
\text { - Statistical uncertainty is } \\
\text { reduced by repeated } \\
\text { measurement }\end{array}$ & $\begin{array}{l}\text { - Image is highly } \\
\text { diffracted } \\
\text { - To improve the speed of } \\
\text { measurement to improve } \\
\text { upon point-by-point } \\
\text { operation }\end{array}$ \\
\hline $\begin{array}{l}\text { [3] Spacer height } \\
\text { adjustment }\end{array}$ & - Polishing of spacers & $\begin{array}{l}\text { - Currently not in-situ, } \\
\text { mirror mount is removed } \\
\text { - Polish to } 0.05-0.1 \mu \mathrm{m} \\
\text { precision }\end{array}$ & $\begin{array}{l}\text { New sensor system to } \\
\text { simultaneously gage all } \\
\text { the spacer heights to } 0.1 \\
\mu \mathrm{m}\end{array}$ \\
\hline $\begin{array}{l}\text { [4] Co-alignment of } \\
\text { mirrors for 2-reflection }\end{array}$ & $\begin{array}{l}\text { - mirrors aligned to } \\
\text { optical axis established } \\
\text { by the beam's direction }\end{array}$ & $\begin{array}{l}\text { - Co-alignment to optical } \\
\text { axis is within 1-2" in the } \\
\text { radial and tangential } \\
\text { directions }\end{array}$ & $\begin{array}{l}\text { - Large amount of } \\
\text { material to remove } \\
\text { - Coupling of angular and } \\
\text { translational correction } \\
\text { - Large difference in } \\
\text { sensitivities between } \\
\text { angular and translational } \\
\text { correction }\end{array}$ \\
\hline [5] Bonding of mirrors & - Epoxy application & $\begin{array}{l}\text { - Manual application } \\
\text { - Small error in HPD, } \Delta \text { in } \\
\text { HPD } \simeq+/-0.2^{\prime} \\
\text { - Bonding is stable }\end{array}$ & $\begin{array}{l}\text { - Improve bonding speed } \\
\text { - Extended stability test } \\
\text { - Bonding strength and } \\
\text { other environment tests }\end{array}$ \\
\hline
\end{tabular}

Table 1. Summary of the current process, status and issues of alignment and bonding in the 4-Point mounting scheme.

\section{X-RAY TESTS AND RESULTS}

\subsection{X-ray imaging test}

In the past months, we have bonded many mirrors onto silicon platform in the 4-Point mounting scheme described above; we also brought 3 single pairs to x-ray test. The results are encouraging. In this serious of tests, we have progressively improved from 6.4" to 4" (HPD, full illumination, with an Ti impact source). The x-ray CCD camera was operated in the imaging mode (x-ray events are piled up) and the sub-aperture was taken with a movable mask of about $3^{\circ}$. Even in the very first test of this series---the first one after we departed from the glass mirrors and "edgebonding" method---the focus was very good, but the error was dominated by the relatively large axial figure imperfection, showing itself in the sub-aperture HPD. Nevertheless, this is already a record-setting resolution and it is clear that we entered a different regime. In the second and third test, we have improved the axial figure with ionbeam finished mirrors, and got much better sub-aperture HPD. Figure 5 shows the result of one sector in test \#2 and its dependence on azimuth. (The "relative azimuth" scaled to the physical azimuthal angle by a factor of 1.6; the full 
mirror azimuthal extent is from $-15^{\circ}$ to $+15^{\circ}$.) It is clear that, despite the many highly focus sectors with HPD $\sim 2^{\prime \prime}$, there is a systematic error in the mirrors or the bonding that caused the HPD on one side of the pairs to be larger.)
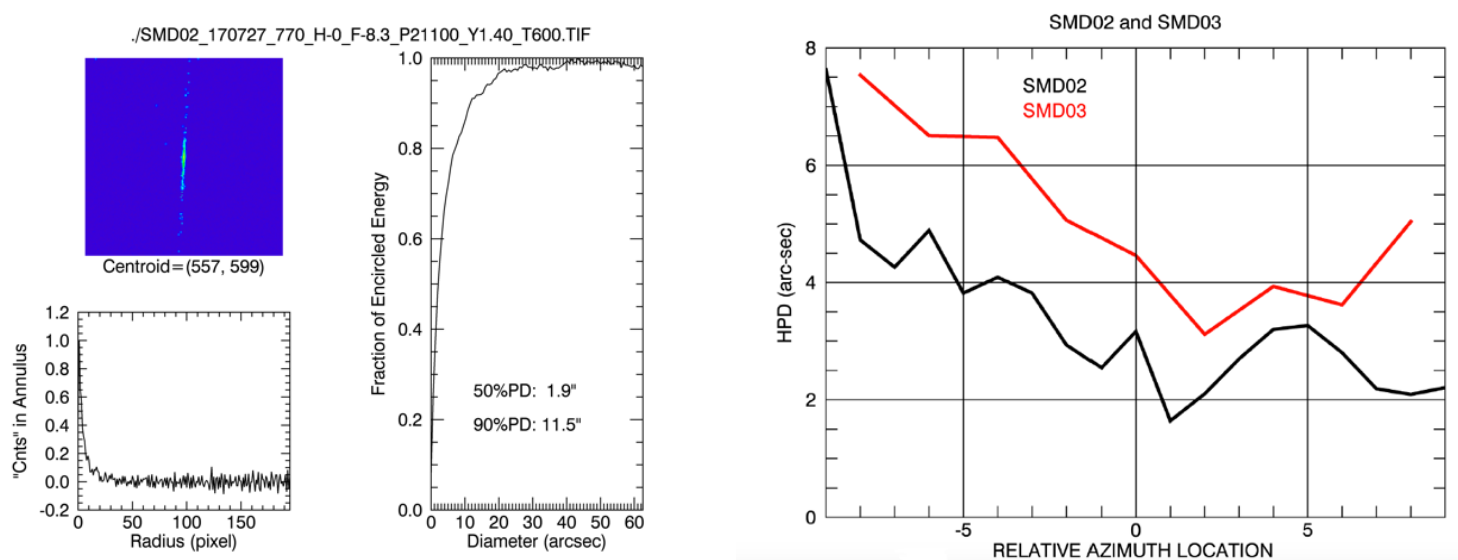

Figure 5. Sub-aperture imaging of the silicon mirrors with ion-beam finishing and were bonded in a 4-point silicon mount. The left panel show the image and the encircled energy function of a sector in test SMD02. The panel on the right shows the HPD as a function of azimuth.

The overall image is also very encouraging. In the latest test, an HPD of 4.5" for a fully illuminated mirror was obtained. The result is shown in Figure 6. Unfortunately, there is still a residual alignment error, mainly of the pitch angle between the primary and the secondary mirror. This is apparent in the distribution of centroids of sector images, shown in the right panel of Figure 6.
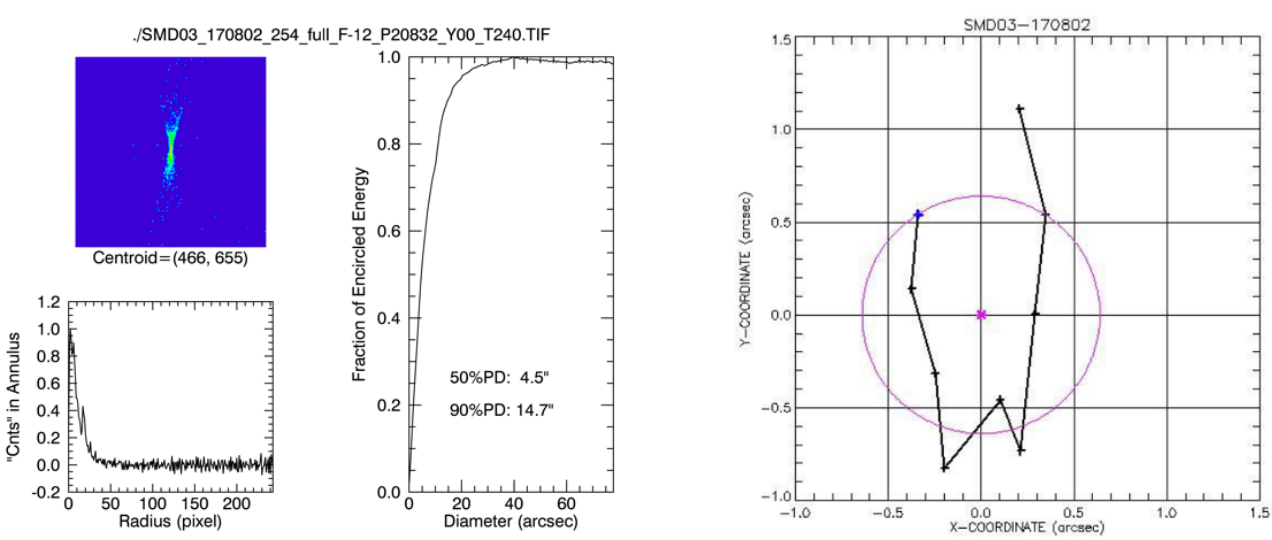

Figure 6. Result from test SMD03. (Left) Encircled energy function and HPD of the mirror pair in full illumination. Silicon mirrors with ion-beam finishing were bonded in a 4-point silicon mount. (Right) The distribution of image centers for sub-apertures. The distribution has an effective focusing error of 1.4" (rms diameter), which is also apparent in the full image on the left panel.

\section{SUMMARY AND PLANS}

\subsection{Summary}

At GSFC, we have developed a full suite of capabilities to fabricate precise silicon mirrors, from slicing, shapeforming, trimming, polishing, to etching. The final ion-beam figuring process correct the axial sag. We also developed a compatible alignment and bonding method to integrate these highly precise silicon mirrors. The process, the 4-Point kinematic mount method, is simple but effective. It represents a departure from our previous "edge-bonding" 
technique. It takes advantage of the stiff silicon mirror and addresses several of the inadequacies of the edge-bonding. The combination of better mirror and mounting technique resulted in much improved resolution in three x-ray tests, each better than the previous. The latest tests showed angular resolution of a fully illuminated pair of mirror at 4 " HPD.

Despite the initial success, there are additional errors that were not accounted for. The resolution of the azimuthal sectors was expected to be $\sim 2$ " from the optical metrology. This is largely borne out by direct x-ray measurement, even though not all sectors were as good as expected, as shown in Figure 5. Clearly, there are either systematic errors on the parts, introduced during the bonding process, or due to other environmental factors such as thermal effects. We have just begun to investigate these possibilities as we run more tests.

\subsection{Plans}

To proceed and advance the technology further, we have planned the following: (1) To improve the current bonding process and better understand the systematic errors and uncertainties. (2) To build a small version of a meta-shell to explore the technical issues at a more integrated level.

To better understand the 4-point alignment process, we will study systematic effects such as thermal effects. Some of the thermal effect may be due to CTE mismatch from small parts on the test platform. Positioning of mirror with absolute control of optical axis position may also needed to be confirmed. One source of error could have been a small deviation of the optical beam from parallelism (the beam is used to set the optical axis location, but it is at a location over $150 \mathrm{~mm}$ away.) We will also stack and bond multiple shells onto existing mirror shells to demonstrate the independence of the current method and to show that there is no stack-up errors. All these can be done with the current set up.

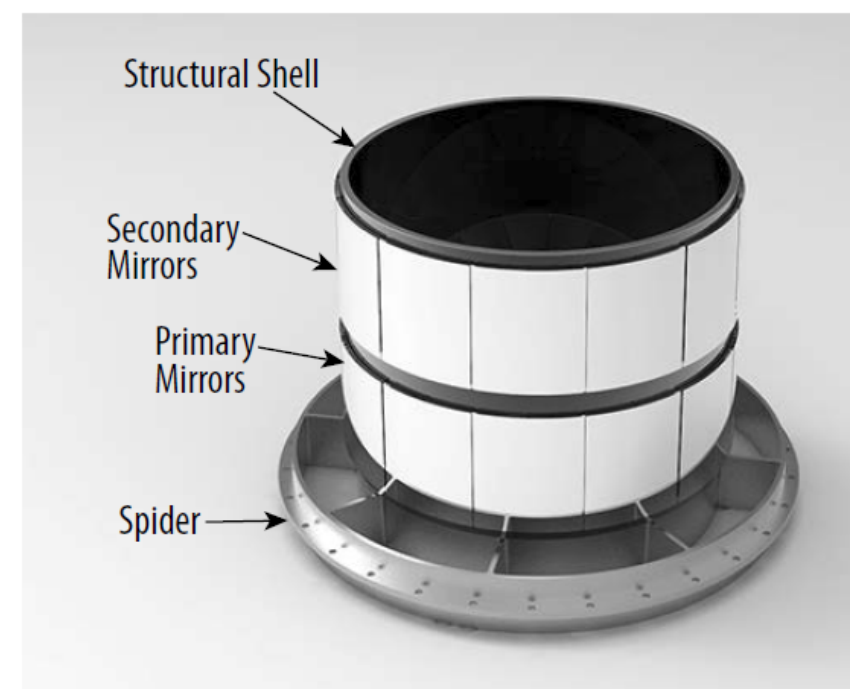

Figure 7. Conceptual structure of a meta-shell.

Furthermore, we plan to build a small meta-shell to explore the issues associated with the meta-shell. In the metashell, the setting of the optical axis will have to be coincided with that of the rotational axis of the meta-shell. The physical rotational axis will have to have small run-out $<1^{\prime \prime}$. The stability of the meta-shell and its alignment need to be stable or recoverable over the course of integration. If that is successful, we plan to perform environmental tests (vibration, shock, acoustic, thermal-vacuum tests) on the shell to improve its technology readiness level. Our goal is to demonstrate a meta-shell having a resolution of $2 "$ - 5" in the $1-10 \mathrm{keV}$ band.

\section{REFERENCES}

[1] S. L. O’Dell, et al., "High resolution x-ray optics," Proc. SPIE 7803, 78030H (2010).

[2] Y. Tanaka, "The Astro-D mission," Adv. Space Res. 10, (2) 255 (1990). 
[3] P. J. Serlemitsos, et al., "The X-ray Telescope onboard Suzaku," Pub. Astron. Soc. Japan 59, S9 (2007).

[4] W. W. Craig, et al., "Fabrication of NuSTAR flight optics," Proc. SPIE 8147, 81470H (2011).

[5] F. A. Harrison, et al., "The Nuclear Spectroscopic Telescope Array (NuSTAR) high-energy x-ray mission," Ap. J. 770, 103 (2013).

[6] T. Takahashi, et al., "The Astro-H x-ray observatory," Proc. SPIE 8443, $84431 \mathrm{Z}$ (2012).

[7] M. Bavdaz, et al., "The Athena optics," Proc. SPIE 9603, 96030J (2015).

[8] R. E. Riveros, et al., "Progress on the fabrication of high resolution and lightweight monocrystalline silicon x-ray mirrors," Proc. SPIE 9905, 990521 (2016)

[9] R. E. Riveros, et al., "Progress on the fabrication of lightweight single crystal silicon x-ray mirrors," Proc. SPIE 10399,10399 (2017)

[10] T. Saha, et al., "Optical design of the STAR-X telescope," Proc. SPIE 10399, 10399 (2017)

[11] R. S. McClelland, et al., "The STAR-X x-ray telescope assembly (XTA)," Proc. SPIE 10399, 10399 (2017)

[12] W. W. Zhang, et al., "Monocrystalline silicon and the meta-shell approach to building x-ray astronomical optics," Proc. SPIE 10399, 10399 (2017)

[13] K. W. Chan, et al., "Aligning, bonding and testing mirrors for lightweight x-ray telescopes," Proc. SPIE 9603, 96030Z (2015)

[14] M. P. Biskach, et al., "Alignment and integration of thin, lightweight x-ray optics into modules," Proc. SPIE 9144, 914446 (2014) 